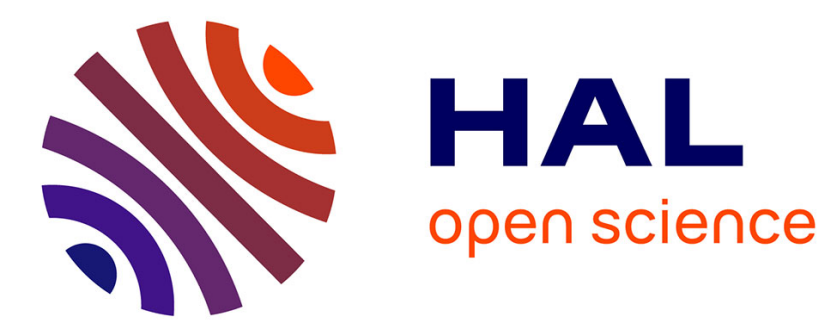

\title{
Long Range Correlations in Quantum Solids
}

Ettore Vitali, Pierantonio Arrighetti, Maurizio Rossi, Davide Emilio Galli

\section{To cite this version:}

Ettore Vitali, Pierantonio Arrighetti, Maurizio Rossi, Davide Emilio Galli. Long Range Correlations in Quantum Solids. Molecular Physics, 2011, pp.1. 10.1080/00268976.2011.610370 . hal-00736795

\section{HAL Id: hal-00736795 \\ https://hal.science/hal-00736795}

Submitted on 30 Sep 2012

HAL is a multi-disciplinary open access archive for the deposit and dissemination of scientific research documents, whether they are published or not. The documents may come from teaching and research institutions in France or abroad, or from public or private research centers.
L'archive ouverte pluridisciplinaire $\mathbf{H A L}$, est destinée au dépôt et à la diffusion de documents scientifiques de niveau recherche, publiés ou non, émanant des établissements d'enseignement et de recherche français ou étrangers, des laboratoires publics ou privés. 


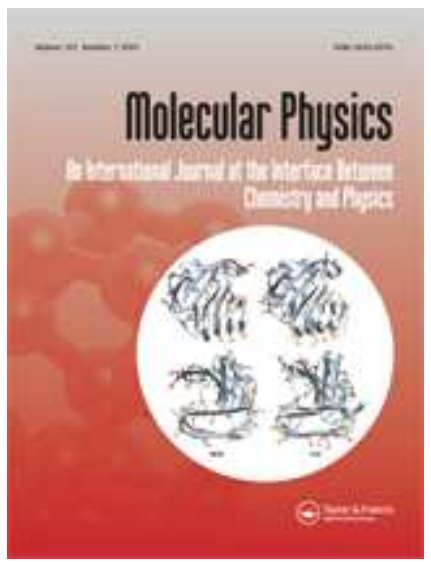

\section{Long Range Correlations in Quantum Solids}

\begin{tabular}{|r|l|}
\hline Journal: & Molecular Physics \\
\hline Manuscript ID: & TMPH-2011-0218.R1 \\
\hline Manuscript Type: & Special Issue in honour of Luciano Reatto \\
\hline Author: & $20-J u l-2011$ \\
\hline Complete List of Authors: & $\begin{array}{l}\text { Vitali, Ettore; Universita' degli Studi di Milano } \\
\text { Arrighetti, Pierantonio; Universita' degli Studi di Milano } \\
\text { Rossi, Maurizio; Politecnico di Milano } \\
\text { Galli, Davide; Universita' degli Studi di Milano, Fisica }\end{array}$ \\
\hline Keywords: & quantum solids, long range correlations, supersolidity \\
\hline & \\
\hline $\begin{array}{l}\text { Note: The following files were submitted by the author for peer review, but cannot be converted } \\
\text { to PDF. You must view these files (e.g. movies) online. }\end{array}$ \\
\hline ART.tex
\end{tabular}

\section{SCHOLARONE Manuscripts}


Molecular Physics

Vol. 00, No. 00, Month 2009, 1-12

\title{
RESEARCH ARTICLE
}

\section{Long Range Correlations in Quantum Solids}

\author{
E. Vitali ${ }^{a}$, P. Arrighetti ${ }^{a}$, M. Rossi ${ }^{b}$, and D.E. Galli ${ }^{a *}$ \\ ${ }^{a}$ Dipartimento di Fisica, Università degli Studi di Milano, via Celoria, 16 - 20133 \\ Milano, Italy. \\ ${ }^{b}$ Dipartimento di Matematica, Politecnico di Milano, Piazza Leonardo da Vinci, 32 - \\ 20133 Milano, Italy \\ (Received 00 Month 200x; final version received 00 Month 200x)
}

\begin{abstract}
Nearly forty years after the admirable paper[1] in which Reatto and Chester suggested a ground state wave function for liquid ${ }^{4} \mathrm{He}$ capturing long-range correlations related to the zeropoint motion of compressional waves (longitudinal phonons), we investigate the possibility of mimicking their approach in the case of solid ${ }^{4}$ He. Starting from elasticity theory, via a "quantization" procedure, we propose a way to describe correlations among particles related to transverse long wavelength oscillations of the crystal lattice. The idea of exploring new kinds of long range correlations in variational models arises from the evidence that some correlations are missing even in the most accurate models, like the Shadow Wave Function, which are not able to describe the long distance behavior of the one-body density matrix in the solid phase computed in a perfect crystal: no variational model of the ground state is known which is able to reproduce qualitatively the long range behavior of this function, which turns out to be an exponential decaying function of the distance when computed via exact finite temperature and zero temperature Path Integral methods. In this paper we derive a functional form for the contribution of the zero-point motion of transverse phonons to the ground state microscopic wave function. Such an approach opens many possibilities of defining variational models, and we propose a first attempt, consisting in a modified Shadow Wave Function.
\end{abstract}

Keywords: Quantum solids; long range correlations, supersolidity

\section{Introduction}

Quantum solids provide a very interesting research field both from a theoretical and from an experimental point of view; they are characterized by a large zero point motion, letting the atoms explore a sizable region in the unit cell of the crystal lattice. This allows quantum statistics to enter the game, giving rise to a scenario suitable for investigating quantum coherence effects in spatially localized systems.

${ }^{4} \mathrm{He}$ at very low temperature, a quite unique pure quantum system, as the external pressure exceeds about $25 \mathrm{~atm}$ becomes a typical example of a quantum solid and a microscopic understanding of the correlations governing its behavior deserves naturally strong interest. Recently Non Classical Rotational Inertia (NCRI) effects have been detected in bulk and confined crystalline ${ }^{4} \mathrm{He}$, a signal that coherence phenomena take place in the system. The experimental findings about the solid phase of ${ }^{4} \mathrm{He}$, in connection with the controversial topic of a possible supersolid

\footnotetext{
*Corresponding author. Email: Davide.Galli@unimi.it
} 
state of matter (i.e. superfluidity taking place in the crystalline phase) can be found in many excellent reviews[2-6].

From the theoretical side, the strong correlations among Helium atoms make Quantum Monte Carlo (QMC) methods the most powerful technique to study the behavior of such a system. Since ${ }^{4} \mathrm{He}$ atoms obey Bose statistics, QMC are able to provide exact estimations of equilibrium properties, relying on imaginary time Schrödinger equation. As far as coherence phenomena are concerned, it has been possible to show that in a crystalline commensurate sample of ${ }^{4} \mathrm{He}$ Bose Einstein Condensation (BEC) does not take place, a result that had been initially obtained at finite temperature [7, 8] and, afterwords, has been confirmed also at $T=0 \mathrm{~K}[5,9]$, preventing the possibility of a phase transition in the perfect crystal taking place at $T \leq 0.2 \mathrm{~K}$.

The formalism of exact Path Integral QMC methods, both a finite and at zero temperature, is made of multidimensional integrals involving propagators and this prevents the possibility of keeping under control the physical correlations underlying the observed properties. Nevertheless, among the several interesting features of QMC, during last decades such methods have provided the possibility to build up and test variational approximations for the Ground State wave function, comparing their predictions with exact ones. This opens the possibility to put on a firm ground the microscopic comprehension of the behavior of the system: an accurate variational model explicitly contains the physical interatomic correlations underlying the rich phenomenology of ${ }^{4} \mathrm{He}$ systems.

The simplest way to build up a variational model of solid Helium is to correct a traditional Jastrow correlation term $\phi_{J}(\mathcal{R})$ :

$$
\phi_{J}(\mathcal{R})=\prod_{1 \leq i<j \leq N} \exp \left(-u\left(\left|\vec{r}_{i}-\vec{r}_{j}\right|\right)\right), \quad \mathcal{R} \equiv\left(\vec{r}_{1}, \ldots, \vec{r}_{N}\right)
$$

via an harmonic particle-site interaction introducing a priori a crystal lattice $\left\{\vec{R}_{i}\right\}$, obtaining the Jastrow-Nosanow wave function[10-12]:

$$
\psi_{J N}(\mathcal{R})=\phi_{J}(\mathcal{R}) \prod_{i=1}^{N} \exp \left(-\alpha\left|\vec{r}_{i}-\vec{R}_{i}\right|^{2}\right)
$$

This model has the disadvantage that it breaks Bose symmetry, preventing automatically the possibility of describing coherence phenomena. One possible way to recover the Bose symmetry is provided by the Shadow Wave Function (SWF)[13], where the Jastrow term is improved via an harmonic coupling of Helium atoms positions, the real variables, with additional shadow variables $\mathcal{S}=\left(\vec{s}_{1}, \ldots, \vec{s}_{N}\right)$, which are subsequently integrated over:

$$
\psi_{s h}(\mathcal{R})=\phi_{J, r}(\mathcal{R}) \int d \mathcal{S} \prod_{i=1}^{N} \exp \left(-c\left|\vec{r}_{i}-\vec{s}_{i}\right|^{2}\right) \phi_{J, s}(\mathcal{S})
$$

The label in $\phi_{J, r, s}$ means that the pseudopotential $u(r)$ among real variables is different from the one among shadows. The SWF is Bose symmetric and translationally invariant, features shared by the exact Ground State wave function[14], and describes the solid phase as a result of a spontaneous translational symmetry 
breaking mechanism: when the density is above a certain value the many-body correlations present in the SWF become so strong that the solid phase is stable and its energy is below the energy of the metastable liquid phase. SWF still provide the most accurate variational description of liquid and solid ${ }^{4} \mathrm{He}$ with an accurate prediction of freezing and melting densities[15]: the energy per particle obtained with a fully optimized SWF turns out to be no more than $3 \%$ above the one computed with "exact" QMC methods and with an almost constant gap in a wide density range, leading to a very accurate equation of state.

Although SWF has been able to provide an extremely accurate description of many properties of liquid and solid Helium systems[16-24], in the solid phase it predicts $[25,26]$ Bose Einstein Condensation (BEC) which, for a perfect crystal at $T=0 \mathrm{~K}$, is not confirmed by exact calculations [5, 9]. This suggests that some important physical correlations are not present at the SWF level of the theory. As suggested also in Ref.[8], we argue that such correlations are long-range, consistently with the behavior of the exact One Body Density Matrix (OBDM) $\rho_{1}\left(\vec{r}-\vec{r}^{\prime}\right)$, which remains non-zero for distances much larger than the lattice spacing, before decaying exponentially as $\left|\vec{r}-\vec{r}^{\prime}\right| \rightarrow \infty$.

In a admirable paper about liquid ${ }^{4} \mathrm{He}$ [1], Reatto and Chester performed an hydrodynamic analysis of large scale dynamics of Helium, relying on a "quantization" of classical sound field, and provided a mathematical model describing zero-point motion of density fluctuations: compressional waves (longitudinal phonons). Such a zero-point dynamics corresponds to collective motions of the atoms in the Bose fluid, resulting in a long-range two-body interparticle correlation of the form:

$$
u_{l r}(r) \propto \frac{1}{r^{2}+a^{2}}
$$

Such a correlation has been shown to improve the variational description, in particular correcting the small wave vector limit of the static structure factor (see Fig.1), but still is not able to account for the suppression of the condensate fraction in the crystalline phase[1,27].

The aim of this work is to improve the variational model for the wave function in the solid phase, where also transverse phonons exist and the exact ground state has to contain their zero-point motion, resulting in additional long-range correlations. Our idea has been to mimic the procedure in [1] in the solid phase, relying on elasticity theory. Starting from continuum mechanics, we have defined a quantum mechanical operator which adds the zero-point motion of transverse modes to a given wave function. Under a "small currents" approximation we have obtained a possible form for the resulting long-range interatomic correlations and inserted them into a SWF-like model. From variational calculations we realized that our correction makes the condensate fraction decrease with respect to SWF results, but it is still non vanishing, making interesting in the future to study different ways of exploiting the "quantum elasticity" approach we have here introduced.

This paper is structured as follows: in the next section, after a review of ReattoChester approach, we will present the methodology of "quantization" of transverse dynamics in an elastic continuum, providing a new Shadow Wave function containing zero point motion of transverse phonons; in Sec. III, we will present variational Monte Carlo calculations relying on the new wave function and discuss possible future perspectives. 


\section{Methodology}

\subsection{Reatto-Chester theory}

As a first step, we find useful to briefly sketch the derivation of the result (4), which will provide a guide for our analysis. The idea is to start from an "hydrodynamic Hamiltonian" in the local density variable $\rho(\vec{r}, t)$ of the fluid, at lowest order in deviations from global thermal equilibrium:

$$
\mathcal{E}(\{\vec{v}(\vec{r}, t)\},\{\rho(\vec{r}, t)\})=\frac{1}{2} m \rho \int d \vec{r}|\vec{v}(\vec{r}, t)|^{2}+\frac{1}{2 \mathcal{K}_{T} \rho^{2}} \int d \vec{r}(\rho(\vec{r}, t)-\rho)^{2}
$$

In $(5) \vec{v}(\vec{r}, t)$ is the velocity field of the continuum and the prefactor of the potential energy derives from standard equilibrium thermodynamic relations between $\frac{\partial^{2} \mathcal{E}}{\partial \rho^{2}}$ and the isothermal compressibility $\mathcal{K}_{T}$. The linearized form of the continuity equation in wave vector space:

$$
\frac{\partial \rho_{\vec{q}}(t)}{\partial t}=i \rho \vec{q} \cdot \vec{v}_{\vec{q}}(t)
$$

where it is transparent that we are dealing with longitudinal dynamics, together with (5), makes straightforward to obtain an Hamiltonian describing a gas of harmonic oscillators for the degrees of freedom $\left\{\rho_{\vec{q}}\right\}$, Fourier components of the local density. Interpreting (5) as a quantum Hamiltonian, $\left\{\rho_{\vec{q}}\right\}$ playing the role of position operators (one for each wave vector), we get immediately a ground state wave function of the form:

$$
\Psi_{0}\left(\left\{\rho_{\vec{q}}\right\}\right) \propto e^{-\frac{m s}{2 N \hbar} \sum_{\vec{q}} \frac{\left|\rho_{\vec{q}}\right|^{2}}{|\vec{q}|}}
$$

$s$ being the longitudinal sound velocity. The link with many body physics arises naturally from the definition of the density fluctuation operator:

$$
\hat{\rho}_{\vec{q}}=\sum_{i=1}^{N} e^{-i \vec{q} \cdot \overrightarrow{\hat{r}}_{i}}
$$

which maps (7) into a simple multiplication operator in the Hilbert space of the Helium sample: the strategy is to apply such an operator to a given model of the ground state wave function of the Helium system in order to "correct" it by "adding" the zero-point motion of longitudinal phonons. Performing the sum over the wave vectors in the continuum limit via the introduction of a suitable ultraviolet cutoff we get a two-body Jastrow wave function with a pseudopotential precisely of the form (4).

Such a term has been proved to improve the variational SWF description, the most important novelty being the accuracy of the low wave vector limit of the static structure factor of the system (see Fig.1); despite that, exact analytical results indicate that BEC survives in the model [1], so one has to look for other motivations to explain the vanishing BEC fraction resulting from exact calculations. 


\subsection{Elasticity and transverse modes}

Here we try to devise an analogous strategy for transverse modes in the crystalline phase, starting from classical elasticity theory (for a general review many excellent textbooks exist, for example Ref. [28]). Let's start considering an elastic medium described in terms of a displacement field $\vec{u}(\vec{r}, t)$, a dynamical variable physically representing deformations with respect to a given equilibrium configuration; in the language of continuum mechanics, deformations give rise to a stress field in the sample generating internal forces described by the stress tensor $T_{\alpha, \beta}(\vec{r}, t)$. The equations of motion of the medium, in the infinitesimal deformations regime, may be written as:

$$
\rho \frac{\partial^{2} u_{\alpha}(\vec{r}, t)}{\partial t^{2}}=\sum_{\beta=1}^{3} \frac{\partial T_{\beta, \alpha}(\vec{r}, t)}{\partial r_{\beta}}+b_{\alpha}(\vec{r}, t)
$$

where $\vec{b}(\vec{r}, t)$ represents external fields, volume forces acting on the system. In the linear approximation, under the assumption of homogeneity and isotropy, ${ }^{1}$ symmetry arguments lead to the following constitutive equation:

$$
T_{\alpha, \beta}(\vec{r}, t)=\lambda \delta_{\alpha, \beta} \vec{\nabla} \cdot \vec{u}(\vec{r}, t)+\mu\left(\frac{\partial u_{\alpha}(\vec{r}, t)}{\partial r_{\beta}}+\frac{\partial u_{\beta}(\vec{r}, t)}{\partial r_{\alpha}}\right)
$$

$\lambda$ and $\mu$ being phenomenological constants, the Lamé coefficients. Putting together (9) and (10) we arrive to the famous Navier equation for elastic media:

$$
\rho \frac{\partial^{2} u_{\alpha}(\vec{r}, t)}{\partial t^{2}}=(\lambda+\mu) \frac{\partial}{\partial r_{\beta}}(\vec{\nabla} \cdot \vec{u}(\vec{r}, t))+\mu \sum_{\beta=1}^{3} \frac{\partial^{2} u_{\alpha}(\vec{r}, t)}{\partial r_{\beta}^{2}}+b_{\alpha}(\vec{r}, t)
$$

Focusing on the dynamics of transverse displacement fields, defined by:

$$
\vec{\nabla} \cdot \vec{u}(\vec{r}, t)=0
$$

if all the external forces vanish, we get the simple equation:

$$
\rho \frac{\partial^{2} u_{\alpha}(\vec{r}, t)}{\partial t^{2}}=\mu \sum_{\beta=1}^{3} \frac{\partial^{2} u_{\alpha}(\vec{r}, t)}{\partial r_{\beta}^{2}}
$$

which, if we are allowed to consider, at first order, the medium as incompressible $\rho=$ cost, is simply a D'Alembert equation describing waves propagating at velocity $v_{T}=\sqrt{\frac{\mu}{\rho}}$. The transverse contribution to the elastic energy of the continuum

\footnotetext{
${ }^{1}$ To be precise, the crystalline structure hcp of Helium samples at $T=0$ and $P \simeq 25$ bars is not isotropic; nevertheless we are making a "coarse-grained" large scale description, and, at a preliminary level, we make an isotropy hypothesis, but a generalization could be straightforward.
} 
medium may be written as follows:

$$
\mathcal{E}\left(\left\{\vec{j}_{\vec{q}}\right\},\left\{\vec{u}_{\vec{q}}\right\}\right)=\frac{1}{V} \sum_{\vec{q}}\left(\frac{\vec{j}_{\vec{q}} \cdot \vec{j}_{-\vec{q}}}{2 \rho}+\frac{1}{2} \mu|\vec{q}|^{2} \vec{u}_{\vec{q}} \cdot \vec{u}_{-\vec{q}}\right)
$$

where

$$
\vec{j}(\vec{r}, t)=\rho \frac{\partial \vec{u}(\vec{r}, t)}{\partial t}=\frac{1}{V} \sum_{\vec{q}} e^{i \vec{q} \cdot \vec{r}} \vec{j}_{\vec{q}}(t)
$$

is the local current density and $\vec{u}_{\vec{q}}$ and $\vec{j}_{\vec{q}}$ are the Fourier component of the displacement field and of the local current respectively, with

$$
\vec{q} \cdot \vec{j}_{\vec{q}}(t)=0=\vec{q} \cdot \vec{u}_{\vec{q}}(t)
$$

to fulfill the transversality condition.

In (14) the local current plays the role of the linear momentum in the harmonic oscillators, of "mass" $\rho$ and "elastic constant" $\mu|\vec{q}|^{2}$. Now, in classical mechanics, minimum energy would naturally correspond to vanishing of the currents, but, from a quantum point of view, we may expect a zero-point motion governed by a "wave function" either in the "displacement field variable" or in the "current variable". It is evident now that our result is completely analogous to the hydrodynamic one discussed above, the only difference being that now the basic dynamical degree of freedom is the displacement field. While $\vec{u}(\vec{r}, t)$ has no natural counterpart in a many body microscopic theory, the local current has a well known expression, so that we are induced to use the "momentum representation". In the basic harmonic oscillator with Hamiltonian:

$$
\frac{\hat{p}^{2}}{2 m}+\frac{1}{2} k \hat{x}^{2}
$$

the ground state in momentum representation is given by:

$$
c_{0}(p) \propto e^{-\frac{p^{2}}{2 \hbar \sqrt{k m}}}
$$

so that we are induced to write the ground state of (14) in "current representation" in the following way:

$$
\Psi_{0}\left(\left\{\vec{j}_{\vec{q}}\right\}\right) \propto \exp \left(-\sum_{\vec{q}} \frac{\vec{j}_{\vec{q}} \cdot \vec{j}_{-\vec{q}}}{2 \hbar \sqrt{\rho \mu}|\vec{q}|}\right)
$$

How can we use now (19) to "correct" a given variational model for a collection of $N$ bosons in the crystalline phase?

In order to clarify the problem we face, let's consider for a while the simple one-dimensional Hamiltonian (17), and suppose we have constructed two different "variational models", pretending not to know the exact ground state: 


$$
\phi_{1}(x) \propto 1 \quad \phi_{2}(x) \propto \delta(x)
$$

Of course both $\phi_{1}(x)$ and $\phi_{2}(x)$ do not describe the correct zero-point motion, the first one because it is "too delocalized", lacking confinement, while the latter because it is "frozen", and has to be somehow delocalized. How can we correct? The idea is the following: the classical variables $x$ and $p$ display simple oscillatory dynamics and then we are induced to "quantize" their zero point motion via expressions of the form $e^{-a x^{2}}$ and $e^{-b p^{2}}$, precisely as it has been done for the local density in the description of longitudinal motion, but what does it mean? In the quantum mechanics formalism, we define two operators of the form:

$$
\hat{A}_{1} \propto e^{-a \hat{x}^{2}} \quad \hat{A}_{2} \propto e^{-b \hat{p}^{2}}
$$

acting on (20). It is easy to check that what we obtain is:

$$
\hat{A}_{1} \phi_{1}(x) \propto e^{-a x^{2}} \quad \hat{A}_{1} \phi_{2}(x) \propto \phi_{2}(x) \quad \hat{A}_{2} \phi_{1}(x) \propto \phi_{1}(x) \quad \hat{A}_{2} \phi_{2}(x) \propto e^{-c x^{2}}
$$

We observe that $\hat{A}_{1}$ provides the desired result when it acts on $\phi_{1}(x)$, but it doesn't have any effect on $\phi_{2}(x)$; on the other hand the role of $\hat{A}_{2}$ is completely the opposite. So, in some sense, if we use the operator $\hat{A}_{2}$ we have to act on a function which is "too localized" so that it may suitably "add" zero-point motion.

Now, keeping these observations in mind, let's come back to solid Helium: in a many-body first principles quantum model, we may define the local current density operator of the system, whose Fourier components are defined by:

$$
\overrightarrow{\hat{j}}_{\vec{q}}=\frac{1}{2 m} \sum_{i=1}^{N}\left(\left(-i \hbar \frac{\partial}{\partial \vec{r}_{i}}\right) e^{-i \vec{q} \cdot \vec{r}_{i}}+e^{-i \vec{q} \cdot \vec{r}_{i}}\left(-i \hbar \frac{\partial}{\partial \vec{r}_{i}}\right)\right)
$$

The transverse components are given by:

$$
\overrightarrow{\hat{j}}_{\vec{q}}^{T}=\overrightarrow{\hat{j}}_{\vec{q}}-\left(\frac{\vec{q}}{|\vec{q}|} \cdot \overrightarrow{\hat{j}}_{\vec{q}}\right) \frac{\vec{q}}{|\vec{q}|}
$$

and from $(23)$ it is possible to construct the operator:

$$
\hat{\mathcal{J}} \stackrel{\text { def }}{=} \exp \left(-\gamma \sum_{\vec{q}} \frac{1}{|\vec{q}|} \vec{j}_{\vec{q}}^{T} \cdot \overrightarrow{\hat{j}}_{-\vec{q}}^{T}\right) \quad \gamma \geq 0
$$

which plays the same role as $\hat{A}_{2}$ defined above. The similarity to the hydrodynamic approach is transparent. We have learned that we may hope that $\hat{\mathcal{J}}$ could provide the desired result only if we apply it to a model wave function which is somehow "too localized". So, as a preliminary step, a natural idea appears to be that of trying to act on an element of the Hilbert space of the system of the Hartree form: 


$$
\phi\left(\vec{r}_{1}, \ldots, \vec{r}_{N}\right) \propto e^{-\sum_{k=1}^{N} \alpha\left|\vec{r}_{k}-\vec{R}_{k}\right|^{2}}
$$

where $\left\{\vec{R}_{k}\right\}$ are sites of a crystal lattice. The technical difficulty of applying (25) is formidable, and we decide to work at first order in the exponent, relying on the following expression:

$$
\left(\overrightarrow{\hat{j}}_{\vec{q}}^{T} \cdot \overrightarrow{\hat{j}}_{-\vec{q}}^{T}\right) f=-\frac{\hbar^{2}}{m^{2}} \sum_{i, l=1}^{N}\left(e^{-i \vec{q} \cdot\left(\vec{r}_{i}-\vec{r}_{l}\right)} \sum_{\alpha, \beta=1}^{3}\left(\delta_{\alpha, \beta}-\frac{q_{\alpha} q_{\beta}}{|\vec{q}|^{2}}\right) \frac{\partial^{2} f}{\partial r_{i, \alpha} \partial r_{l, \beta}}\right)
$$

which holds for any element $f\left(\vec{r}_{1}, \ldots, \vec{r}_{N}\right)$ such that the r.h.s. of (27) does make sense. The derivation of (27), though a bit lengthy, is a straightforward application of the definitions (23) and (24). Performing the second derivative appearing in (27) when $f$ is $\phi$ defined in (26), and remembering the definition of the cross product it is quite simple to show that:

$$
\sum_{\vec{q}} \frac{\left(\overrightarrow{\hat{j}}_{\vec{q}}^{T} \cdot \overrightarrow{\hat{j}}_{-\vec{q}}^{T}\right)}{|\vec{q}|} \phi \propto\left(-\sum_{i, l=1}^{N} \sum_{\vec{q}} \frac{e^{-i \vec{q} \cdot\left(\vec{r}_{i}-\vec{r}_{l}\right)}}{|\vec{q}|}\left(\hat{q} \times\left(\vec{r}_{i}-\vec{R}_{i}\right)\right) \cdot\left(\hat{q} \times\left(\vec{r}_{l}-\vec{R}_{l}\right)\right)\right) \phi
$$

$\hat{q}$ being the versor in the direction of $\vec{q}$.

Our "small currents" approximation strategy works as follows:

$$
\hat{\mathcal{J}} \phi \simeq\left(\hat{\mathbb{I}}-\gamma \sum_{\vec{q}} \frac{\left(\overrightarrow{\hat{j}}_{\vec{q}}^{T} \cdot \overrightarrow{\hat{j}}_{-\vec{q}}^{T}\right)}{|\vec{q}|}\right) \phi=(1-(\ldots)) \phi \simeq e^{-(\ldots)} \phi
$$

so that we obtain the following function:

$$
(\hat{\mathcal{J}} \phi)\left(\vec{r}_{1}, \ldots, \vec{r}_{N}\right) \propto e^{-\sum_{k=1}^{N} \alpha\left|\vec{r}_{k}-\vec{R}_{k}\right|^{2}} e^{\gamma \sum_{i, l=1}^{N} \sum_{\vec{q}} \frac{e^{-i \vec{q} \cdot\left(\vec{r}_{i}-\vec{r}_{l}\right)}}{|\hat{q}|}\left(\hat{q} \times\left(\vec{r}_{i}-\vec{R}_{i}\right)\right) \cdot\left(\hat{q} \times\left(\vec{r}_{l}-\vec{R}_{l}\right)\right)}
$$

It is evident that the last term in the exponential introduces a long-range correlation depending on transverse oscillations of atoms around the lattice positions. The expression (30) suggests the following picture: as in the Gaussian model (26) the atoms are still localized around the lattice sites but transverse vibrations of different atoms are no more independent; the new pseudopotential favor a cooperative motion, and this is what we expected from the approach we have followed. (30) is our main result: the Hartree wave function, $\phi$, has been improved with a new microscopic term which describes the coupling between transverse vibrations; We argue that in an accurate model of a quantum solid, long range correlation of the form appearing in (30) should be present. The main difficulty is that we should be able to recover the Bose symmetry. In the following we will present a first attempt to pursue this aim relying on the observation that (26) is formally equivalent to the kernel which, in the SWF picture, localizes the variables to the shadow ones; we are thus induced to substitute it with (30) "inventing" a model of the form: 
Figure 1. Static structure factor $S(q)$ in the liquid phase at the equilibrium density with (filled circles) and without (open circles) the contribution of the zero point motion of longitudinal phonons; error bars are lower than the symbol size. Experimental data[29] (crosses) at low wave vectors are also shown.

$\psi_{t r}(\mathcal{R}) \propto \phi_{r}(\mathcal{R}) \int d \mathcal{S} e^{-\sum_{k=1}^{N} \alpha\left|\vec{r}_{k}-\vec{s}_{k}\right|^{2}} e^{\gamma \sum_{i, l=1}^{N} \sum_{\vec{q}} \frac{e^{-i \vec{q} \cdot\left(\vec{q}_{i}-\vec{s}_{l}\right)}}{|\vec{q}|}\left(\hat{q} \times\left(\vec{r}_{i}-\vec{s}_{i}\right)\right) \cdot\left(\hat{q} \times\left(\vec{r}_{l}-\vec{s}_{l}\right)\right)} \phi_{s}(\mathcal{S})$

in which, of course, all the parameters and the pseudopotentials have to be variationally optimized. To take into account the fact that short range correlation in the SWF model have been already optimized, we have introduced also a cut-off in the $q$ summation in the new term, limiting it to introduce only long range correlations, similarly to what was done in Ref.[1].

\section{Results for a first variational implementation}

Now that we have built up a theoretical ground to face the topic of long range zero-point transverse modes, the next step is the characterization of the properties of such a wave function. Before considering the solid phase we will present some results in the liquid phase obtained using only the long range term arising from longitudinal phonons. In this case the SWF turns out to be

$$
\psi_{t r}(\mathcal{R}) \propto \phi_{r}(\mathcal{R}) \int d \mathcal{S} e^{-\sum_{k=1}^{N} v\left(\left|\vec{r}_{k}-\vec{s}_{k}\right|\right)} e^{\gamma_{l} \sum_{i, l=1}^{N} \sum_{\vec{q}} \frac{e^{-\beta|\vec{q}|}}{|\vec{q}|} e^{-i \vec{q} \cdot\left(\vec{s}_{i}-\vec{s}_{l}\right)}} \phi_{S}(\mathcal{S})
$$

In (32) the cut-off in the $q$ summation has been explicitly written, and this introduces the variational parameter $\beta$. In the liquid phase, we have considered $N=256$ particles in a cubic box at the equilibrium density $\rho=0.0218 \AA^{-3}$, and as functional form we employed the one fully-optimized in Ref.[15]. In the optimization of the variational parameters connected with the long range term arising from longitudinal phonons we found $\beta=4 \AA$ and $\gamma_{l}=0.012 \AA^{\circ}$. The long-range term provides only a $1 \%$ gain in the total energy, as found also previously in Ref.[15], and, as expected, it reproduces the correct linear behavior at small wave vectors of the static structure factor; this is shown in Fig. 1.

We have studied the role of the long-range corrections in the solid phase by considering $N=180$ and $N=360$ particles in a simulation box chosen to fit a regular 
hcp crystal at $\rho=0.0294 \AA^{-3}$. In the ground state variational SWF we have considered long-range correlations related to zero-point motion of both longitudinal and transverse phonons. The optimization of the variational parameters turned out to be quite difficult: by following the steepest descendant direction of the energy as function of the variational parameters we arrived to a set of parameters corresponding to an unstable crystalline phase. This suggested us to follow a different procedure: we have fixed the standard variational parameters in the SWF for the solid phase to those optimized in Ref.[30]; the parameter related to the long-range term of the longitudinal phonons has been simply rescaled with respect to the value in the liquid phase according to the change in the sound velocity: from $s \simeq 248 \mathrm{~m} / \mathrm{s}$ to $s \simeq 471 \mathrm{~m} / \mathrm{s}$ which gives $\gamma_{l}=0.0228 \mathrm{~A}^{-1}$; note, in fact, that the multiplicative constant in the exponent of $(7)$ is proportional to the sound velocity $s$. We have then optimized the parameter $\gamma$, controlling the intensity of the transverse phonon term. No sharp minimum was found, but for values of $\gamma$ in the range $[0,0.016] \mathrm{A}^{-2}$ the energy shows a shallow valley with an almost constant value; we decided to fix $\gamma=0.016 \mathrm{~A}^{-2}$. The crystal described by the new SWF is slightly stiffer than that described by a SWF without the correlations related to the zero-point motion of phonons; this can be inferred from the increased height of the Bragg's peaks in the static structure factor $S(q)$, plotted in Fig. 2.

We have computed also the one-body density matrix $\rho_{1}$ along the first neighbor direction in order to explore the effects of the long-range corrections on the off-diagonal properties. It is noteworthy that, by including the zero point motion of phonons (longitudinal and transverse), the long distance tail of $\rho_{1}$ is found depressed with respect to the SWF without long-range correlations. The condensate fraction reduces from $n_{0}=(9.8 \pm 1.5) \times 10^{-6}$ to $n_{0}=(4.2 \pm 0.6) \times 10^{-6}$; the contribution of the transverse phonons is about 2.5 times larger than the longitudinal one, and this was measured by computing $\rho_{1}$ by considering separately the two long-range terms. Our results for $\rho_{1}$ are reported in Fig. 3. Despite the quantitative change in the condensate fraction, no qualitative changes in $\rho_{1}(r)$ were found: we were not able to capture the exponential decay of the exact $\operatorname{OBDM}[5,7,8]$. We argue that the weakness of our first variational attempt to include correlations related the zero point motion of transverse phonons is connected with the use of shadow variables to define equilibrium positions of the lattice in (31); this 
Figure 3. One-body density matrix $\rho_{1}(r)$ computed along the first neighbor direction in an hcp ${ }^{4}$ he crystal at $\rho=0.0294 \mathrm{~A}^{-3}$ with (filled symbols) and without (open symbols) the contribution of the zero point motion of longitudinal and transverse phonons.

is correct only on average. Recently a very interesting Bose symmetric JastrowNosanow wave function, not cumbersome from the numerical point of view, has been introduced[31], of the form:

$$
\psi_{B J N}(\mathcal{R})=\phi_{J}(\mathcal{R}) \prod_{I=1}^{N} \sum_{i=1}^{N} g\left(\left|\vec{r}_{i}-\vec{R}_{I}\right|\right)
$$

where $g$ can be a Gaussian or another one-body localization factor. A symmetric Bose model that could be able to better exploit the physics contained in (30) could be built inserting the long-range correlations in (33), where the equilibrium positions of the lattice appear explicitly without breaking the Bose symmetry. We plan to investigate such a possibility in the future.

\section{Conclusions}

Quantum simulation methods have been of the greatest importance in the study of quantum liquids and solids. Notwithstanding the presence of exact simulation methods for the calculation of ground state properties, the variational method, the first historically introduced, continues to attract special interest. It is in fact the only method able to make evident the microscopic structure of the wave function: the role of different correlations on observable properties of the system. Our main result is that, in the solid phase, we are able to deduce a functional form for additional long range correlations related to the zero-point motion of transverse phonons. This has been obtained via an analysis of classical elasticity theory which provides an elastic energy offering the possibility of "quantization" in suitable variables, mimicking the approach of Reatto and Chester in the liquid phase[1]. This opens many possibilities of defining variational models which contain contribution from correlations among transverse vibrations of the lattice; we propose a first attempt, consisting in a modified Shadow Wave Function. The crystal described by this new variational model of solid ${ }^{4} \mathrm{He}$ turns out to be slightly stiffer with higher Bragg's peaks in the static structure factor and with a lower tail in the one-body density matrix, but no qualitative change is found for the long-range behavior of 
$\rho_{1}$. As discussed in the text, we plan to further investigate new variational models with long range correlations coming from the zero-point motion of longitudinal and transverse phonons.

\section{Acknowledgments}

In entering the PhD School in Physics my Supervisor, L. Reatto, was particularly determined in suggesting, as research topic, the study of quantum liquids and solids via quantum Monte Carlo techniques. I (DEG) have to thank his determination: his suggestion led me into a fascinating adventure of scientific research, which lasts to this day.

We would also like to thank L. Reatto for useful discussions.

\section{References}

[1]L. Reatto and G.V. Chester, Phys. Rev. 15588 (1967).

[2] M.W. Meisel, Physica B 178121 (1992).

[3] N.V. Prokof'ev, Adv. Phys. 56, 381 (2007).

[4]S. Balibar and F. Caupin, J. Phys.: Condens Matter 20173201 (2008).

[5]D.E. Galli and L. Reatto, J. Phys. Soc. Jpn., 77, 111010 (2008).

[6]S. Balibar, Nature 464, 176 (2010).

[7]M. Boninsegni, N.V. Prokof'ev and B.V. Svistunov, Phys. Rev. Lett. 96105301 (2006).

[8]B.K. Clark and D.M. Ceperley, Phys. Rev. Lett. 96105302 (2006).

[9] E. Vitali, M. Rossi, F. Tramonto, D.E. Galli and L. Reatto, Phys. Rev. B 77, 180504(R) (2008).

[10] L.H. Nosanow, Phys. Rev. 146120 (1966).

[11] J.H. Hetherington, W.J. Mullin, and L.H. Nosanow, Phys. Rev. 154175 (1967).

[12] J.P. Hansen and D. Levesque, Phys. Rev. 165, 293 (1968).

[13] S.A. Vitiello, K.Runge and M.H. Kalos, Phys. Rev. Lett. 60, 1970 (1988).

[14]E. Vitali, D.E. Galli and L. Reatto, in "Recent Progress in Many-Body Theories" Vol. 11, 251 (World Scientific, 2008).

[15]S. Moroni, D.E. Galli, S. Fantoni and L. Reatto, Phys. Rev. B 58, 909 (1998).

[16]D. E. Galli, E. Cecchetti, and L. Reatto, Phys. Rev. Lett. 77, 5401 (1996).

[17] Michael Sadd, G. V. Chester, and L. Reatto, Phys. Rev. Lett. 79, 2490 (1997).

[18]D. E. Galli, G. L. Masserini, and L. Reatto, Phys. Rev. B 60, 3476 (1999).

[19]D.E. Galli and L.Reatto, J. of Phys.: Condensed Matter 28, 6009 (2000).

[20]D. E. Galli and L. Reatto, Phys. Rev. B 63, 214515 (2001).

[21]D.E. Galli and L.Reatto, J. Low Temp. Phys., 124, 197 (2001).

[22]D. E. Galli and L. Reatto, Phys. Rev. Lett. 90, 175301 (2003).

[23] M. Rossi, M. Verona, D. E. Galli, and L. Reatto, Phys. Rev. B 69, 212510 (2004).

[24] M. Rossi, D. E. Galli, and L. Reatto, Phys. Rev. B 72, 064516 (2005).

[25] L. Reatto and G.L. Masserini, Phys. Rev. B 38, 4516 (1988).

[26]D. E. Galli, M. Rossi, and L. Reatto, Phys. Rev. B 71, 140506 (2005).

[27]L. Reatto, Phys. Rev. 183, 334 (1969).

[28] J.P. Marder, "Condensed Matter Physics" (Wiley, 2000).

[29]R.B. Hallock, Phys. Rev. A 5, 320 (1975).

[30] T. MacFarland, S. A. Vitiello, L. Reatto, G. V. Chester, and M. H. Kalos, Phys. Rev. B 50, 13577 (1994).

[31]C. Cazorla, G.E. Astrakharchik, J. Casulleras, and J. Boronat, New J. Phys. 11, 013047 (2009). 


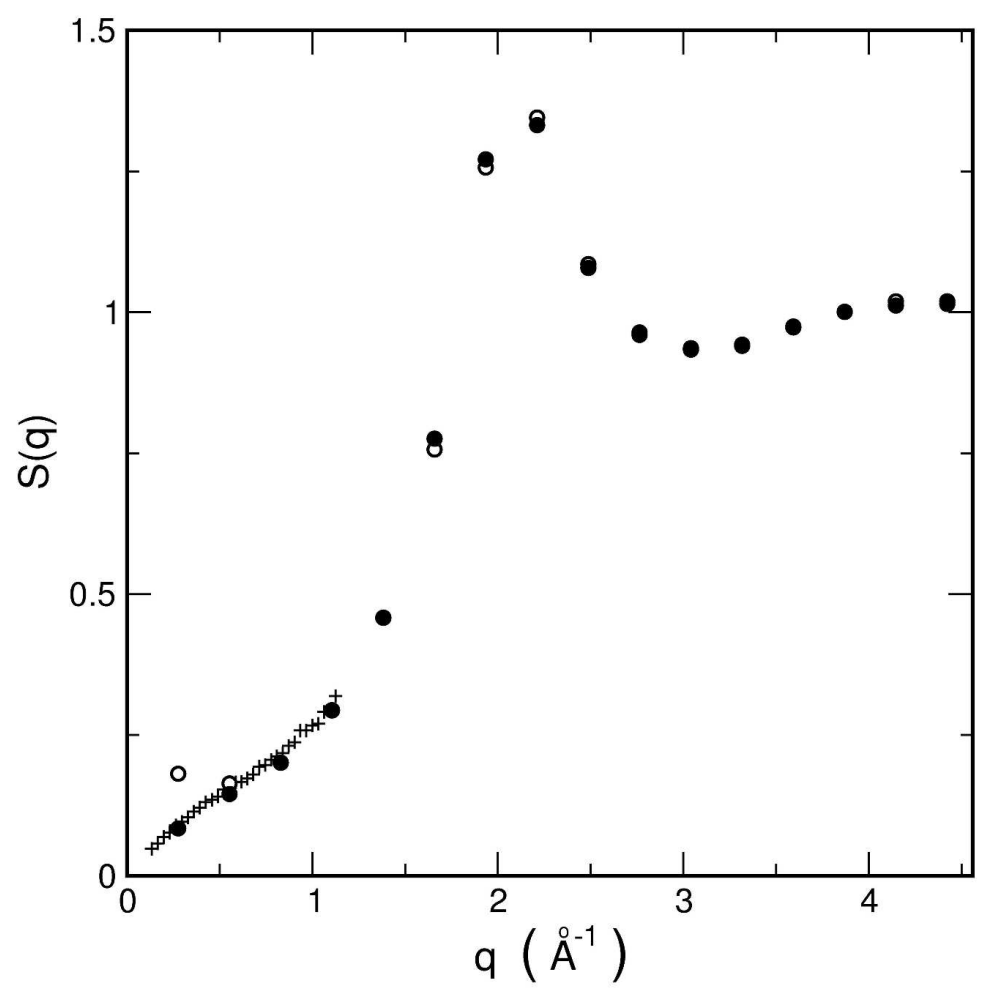

$279 \times 361 \mathrm{~mm}(300 \times 300 \mathrm{DPI})$

URL: http://mc.manuscriptcentral.com/tandf/tmph 


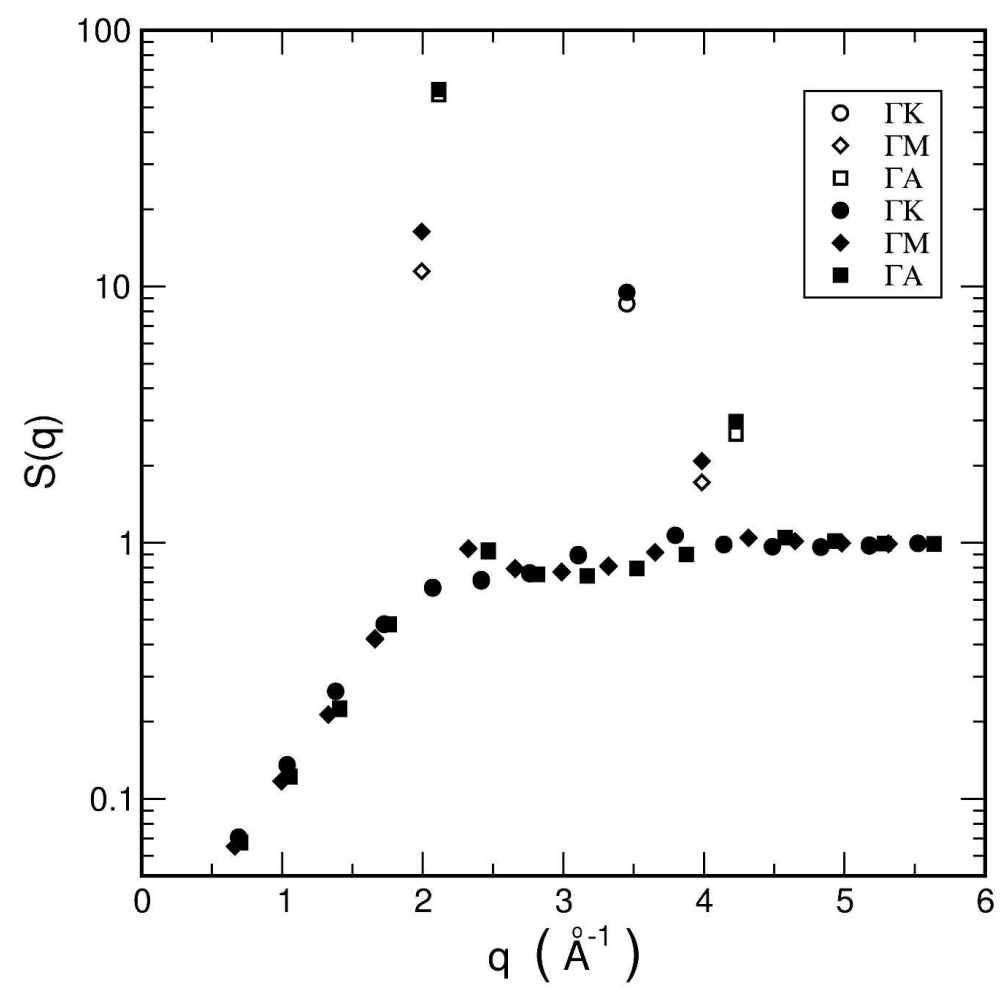

$279 \times 361 \mathrm{~mm}(300 \times 300$ DPI $)$

URL: http://mc.manuscriptcentral.com/tandf/tmph 


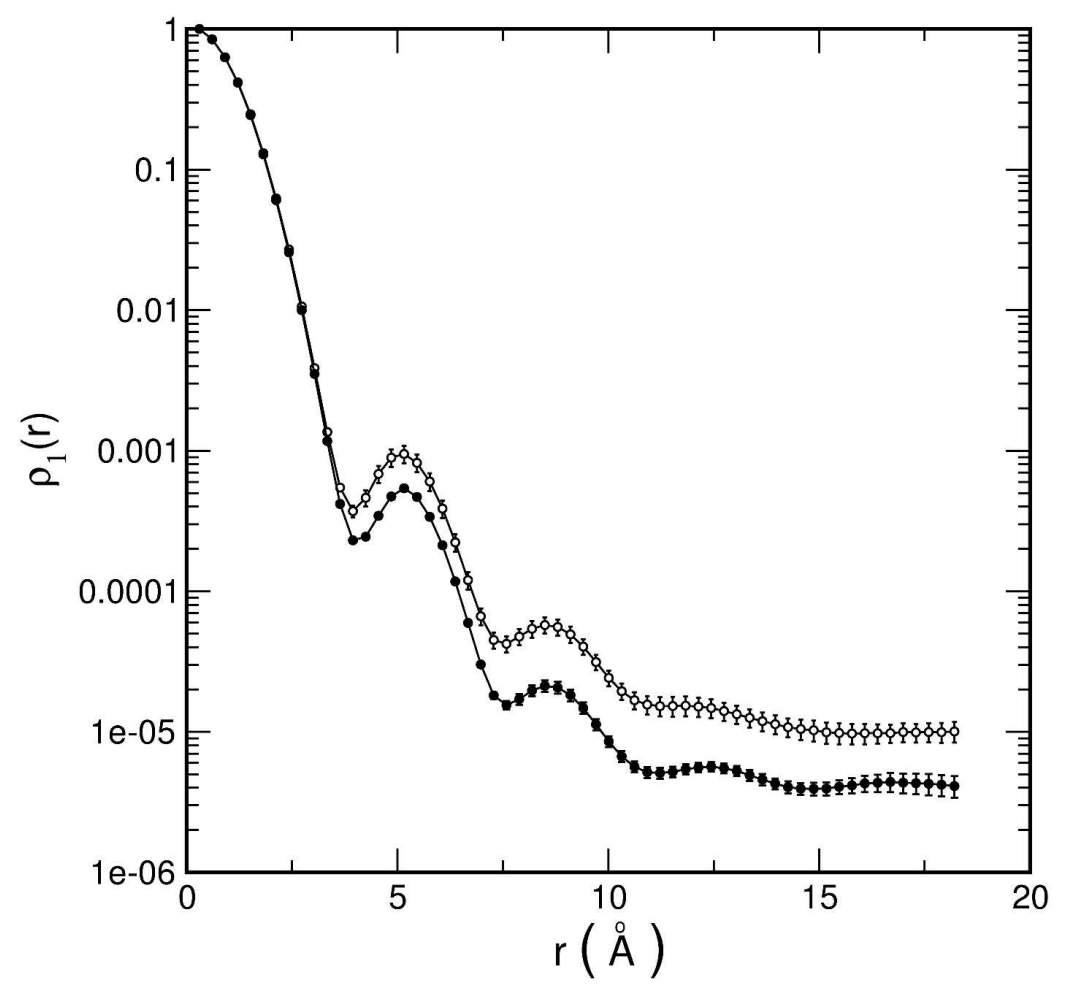

$279 \times 361 \mathrm{~mm}(300 \times 300 \mathrm{DPI})$ 